\title{
Crescimento populacional, ocupação e desemprego dos jovens: a experiência recente da Região Metropolitana de Porto Alegre*
}

\author{
Raul Luís Assumpção Bastos**
}

\begin{abstract}
Este artigo investiga o quanto o tamanho relativo da população juvenil na Região Metropolitana de Porto Alegre (RMPA) afetou a ocupação e o desemprego desse segmento no período 1993-2004. Esta questão ganha relevância no presente tanto pelo fato de que diversos estudos têm apontado a ocorrência do fenômeno de uma onda jovem nas principais regiões metropolitanas do país, nos anos 90, quanto pela correlata observação de uma tendência à elevação do desemprego entre os jovens no período. Assim, primeiramente, é feita uma síntese dos argumentos a respeito dos efeitos do tamanho relativo da coorte juvenil sobre a situação deste grupo populacional no mercado de trabalho. Em seguida, identificam-se as principais tendências da população e da força de trabalho juvenil no mercado de trabalho da RMPA, bem como se estimam os efeitos do tamanho relativo da coorte juvenil sobre a ocupação e o desemprego desse grupo populacional.
\end{abstract}

Palavras-chave: Coorte juvenil. Ocupação juvenil. Desemprego juvenil. Mercado de trabalho metropolitano.

\section{Introdução}

Os jovens constituem um dos grupos populacionais mais afetados pela incidência do desemprego, conforme mostram muitos estudos. Em face dessa constatação, o avanço no conhecimento sobre o seu processo de inserção no mercado de trabalho parece ser condição necessária para enfrentar de forma adequada o fenômeno do desemprego juvenil.

A literatura identifica diversas causas para explicar a situação de maior adversidade relativa dos jovens no mercado de trabalho, entre as quais destacam-se: ausência de experiência anterior de trabalho; formação educacional inadequada; maior sensibilidade do emprego e do desemprego juvenil ao comportamento cíclico das economias; e efeito do tamanho relativo da coorte juvenil sobre seu status no mercado de trabalho.

Este artigo está voltado, fundamentalmente, para a última questão anteriormente referida: trata-se de investigar o quanto a evolução do tamanho relativo da população juvenil, na Região Metropolitana de Porto Alegre (RMPA), afetou a ocupação e o

\footnotetext{
*Trabalho apresentado no XV Encontro Nacional de Estudos Populacionais, Abep, realizado em Caxambu -MG -Brasil, de 18 a 22 de setembro de 2006. Este estudo foi desenvolvido no âmbito do projeto de pesquisa Dimensões da Precarização do Mercado de Trabalho da Região Metropolitana de Porto Alegre, que conta com apoio do CNPq e da FAPERGS. O autor agradece aos colegas Jéferson Daniel de Matos e Maria de Lourdes Jardim pelos comentários e críticas a uma versão preliminar do artigo, bem como pelo apoio da bolsista de iniciação científica da FAPERGS, Thaís Ferreira Persson, na elaboração das tabulações. Erros e omissões por acaso remanescentes são de inteira responsabilidade do autor.

${ }^{\star \star}$ Economista da Fundação de Economia e Estatística e Professor do Departamento de Economia da PUCRS.
} 
desemprego desse grupo populacional no período 1993-2004. A relevância desta questão no presente é evidenciada tanto pelo fato de que diversos estudos (BERCOVICH e MADEIRA, 1990; MUNIZ, 2002; BERCOVICH e MASSÉ, 2004) têm apontado a ocorrência do fenômeno de uma onda jovem 1 no país e em suas principais regiões metropolitanas, nos anos 90 , quanto pela correlata tendência de elevação da incidência do desemprego entre os jovens no período em foco.

\section{Crescimento populacional e os efeitos do tamanho relativo da coorte sobre a situação dos jovens no mercado de trabalho}

O ritmo de crescimento populacional é um dos fatores que determinam o tamanho da força de trabalho de uma economia. Nesse sentido, a dinâmica demográfica pode se constituir em elemento que contribui para pressionar o mercado de trabalho, pois um crescimento populacional elevado requer maior capacidade de geração de emprego pela economia para absorver produtivamente as pessoas que ingressam no mercado de trabalho.

No caso específico da população jovem, ${ }^{2}$ seu ritmo de crescimento e tamanho relativo são recorrentemente apontados como fatores que influenciam suas condições de inserção no mercado de trabalho, nas diferentes experiências nacionais (KORENMAN e NEUMARK, 1997; O'HIGGINS, 1997; OIT, 2000). Ou seja, o crescimento acentuado da população jovem em determinados períodos e o aumento do tamanho da respectiva coorte constituir-se-iam em potenciais agravantes das suas dificuldades de inserção no mercado de trabalho. Em consonância com essa compreensão, uma das hipóteses formuladas pela literatura para explicar a situação dos jovens no mercado de trabalho está, justamente, vinculada à expansão do tamanho da sua coorte (KORENMAN e NEUMARK, 1997; O'HIGGINS, 1997; MUNIZ, 2002). Assim, a hipótese da expansão da coorte (cohort crowding hypothesis) propugna que o aumento do tamanho relativo da população juvenil exerce efeitos adversos sobre o emprego e o desemprego desse grupo populacional no mercado de trabalho. Dessa forma, espera-se que a ocorrência desse fenômeno, em determinados períodos, venha a deteriorar a situação desse grupo populacional no mercado de trabalho.

No que diz respeito à experiência dos países da OCDE, os trabalhos revistos por Korenman e Neumark (1997) indicam a validade desta hipótese. De acordo com a síntese de resultados desses estudos proposta pelos autores, "Parecem existir evidências de um efeito adverso do tamanho da coorte sobre o desemprego, o emprego e os salários juvenis, através de inúmeros países" (KORENMAN e NEUMARK, 1997, p. 7).

Esses autores também desenvolveram uma investigação própria sobre este tema, referente a uma amostra de 15 países da OCDE, para o período de 1970 a 1994. Inicialmente, por meio de uma análise gráfica individualizada dos países, eles concluíram, tentativamente, que:

Primeiro, as taxas de desemprego juvenis parecem responder às mudanças no tamanho relativo da coorte juvenil na forma predita pela hipótese da expansão da coorte. Por outro lado, as taxas de emprego juvenis parecem, pelo menos algumas vezes, moverem-se na direção oposta, caindo enquanto o tamanho relativo da coorte declina, ou não são relacionadas com o tamanho relativo da coorte (KORENMAN e NEUMARK, 1997, p. 13).

Assim, por meio da análise gráfica, os autores só parcialmente conseguiram encontrar indícios da validade da hipótese de expansão da coorte, em particular no que se refere aos seus efeitos adversos sobre a taxa de desemprego juvenil.

\footnotetext{
${ }^{1}$ Este fenômeno constitui-se em uma descontinuidade demográfica, sendo descrito por Bercovich e Massé (2004, p. 7) como "o efeito das ondas de nascimentos na evolução do número de jovens".

${ }^{2}$ Seguindo o critério da Organização das Nações Unidas, os jovens são aqui compreendidos como o grupo populacional de 15 a 24 anos (UN, 2003).
} 
No âmbito desta mesma pesquisa, foram estimados modelos econométricos com diferentes especificações para testar a hipótese do efeito da expansão da coorte sobre as taxas de desemprego e de emprego dos jovens, tendo os autores adotado o procedimento de montar um painel com os dados de sua amostra de 15 países da OCDE (KORENMAN e NEUMARK, 1997). De acordo com o modelo preferido pelos autores, foi estimada uma elasticidade do efeito da expansão da coorte sobre a taxa de desemprego juvenil de 0,6, estatisticamente significativa, enquanto no caso da taxa de emprego, embora a elasticidade do efeito da expansão da coorte tivesse o sinal esperado, ela era muito pequena $(-0,03)$ e não possuía significância estatística (KORENMAN e NEUMARK, 1997, p. 18). Ou seja, os resultados econométricos corroboraram a análise gráfica desenvolvida anteriormente pelos autores de forma individualizada para os países.

O estudo de Korenman e Neumark (1997) também segmentou a população jovem por sexo, com o propósito de analisar a possibilidade de existência de diferenças do efeito da expansão da coorte entre os dois segmentos. De acordo com os resultados dos modelos estimados pelos autores, as mulheres jovens evidenciaram uma maior elasticidade da expansão da coorte sobre sua taxa de desemprego, comparativamente à dos homens. Este resultado os levou a concluir que:

as mulheres jovens suportam um peso desproporcional do desemprego quando a coorte juvenil é maior. Nossa interpretação desse resultado é que os empregadores tendem a contratar os homens jovens primeiro, e voltam-se para as mulheres jovens quando as condições de oferta são limitadas (KORENMAN e NEUMARK, 1997, p. 20).

No tratamento do tema que é objeto de investigação neste artigo, é preciso assinalar que, nas últimas duas décadas do século 20 , ocorreu um pequeno declínio da proporção de jovens em relação à população mundial, passando de 19\% em 1980 para 17,5\% em 2000 (OIT, 2000, p. 4; UN, 2003, p. 76). Esta redução foi um fenômeno que se deu de forma generalizada no âmbito internacional, com exceção da África (OIT, 2000 , p. 4). Não obstante essa tendência comum, deve-se ter presente que, conforme o nível de renda dos países, é diferenciado o tamanho relativo da população juvenil em comparação à população total. Assim, nos países de renda mais baixa, os jovens correspondiam, em 2000, a 19,5\% da população e, naqueles de renda mais alta, a somente $13,2 \%$ (UN, 2003, p. 76). Com isso, pode-se perceber que a pressão demográfica por eles exercida sobre o mercado de trabalho coloca-se de forma distinta de acordo com o nível de desenvolvimento das nações, sendo mais intensa naquelas relativamente mais pobres.

O declínio da proporção de jovens na população constitui uma das dimensões do processo de transição demográfica ${ }^{3}$ nos diferentes países. A esse respeito, no que se refere especificamente à experiência latino-americana, o Celade ${ }^{4}$ propôs a seguinte classificação dos países quanto ao estágio desse processo (DIEZ DE MEDINA, 2001, p. 16-17):

- transição avançada - compreende os países que registram taxas de crescimento de suas populações de $1,0 \%$ ao ano ou inferior, mortalidade infantil moderada ou baixa e um grau de urbanização alto. Neste caso encontram-se Argentina, Chile, Cuba e Uruguai;

- plena transição - corresponde aos países que evidenciam taxas de crescimento populacional de cerca de $2,0 \%$ ao ano e taxas de mortalidade e de natalidade infantis em declínio. Aqui estão incluídos Brasil, Colômbia, Costa Rica, Equador, México, Panamá, Peru, República Dominicana e Venezuela;

- transição moderada - nesta situação encontram-se países com taxas de

\footnotetext{
${ }^{3}$ A transição demográfica é definida como “[...] um processo histórico de grande duração [...] cujo núcleo é o descenso sustentado da fecundidade e da mortalidade" (CEPAL, 2004, p. 34).

${ }^{4}$ Centro Latinoamericano y Caribenho de Demografía, Divisão de População da Cepal.
} 
crescimento populacional de $3,0 \%$ ao ano, que apresentam mortalidade infantil em queda e uma população jovem com natalidade elevada, além de grande proporção da população no meio rural. Neste grupo estão El Salvador, Guatemala, Honduras, Nicarágua e Paraguai;

- transição incipiente - refere-se aos países que apresentam crescimento populacional da ordem de $2,5 \%$ ao ano, com taxas de mortalidade e natalidade infantis muito elevadas e persistentes, grandes proporções de crianças e jovens na população, bem como com a maior parte das suas populações residindo no meio rural. Nesta situação encontram-se a Bolívia e o Haiti.

No que diz respeito à população jovem, seu ritmo de crescimento vem se desacelerando na América Latina desde os anos 70 (DIEZ DE MEDINA, 2001, p. 17). Assim, enquanto na primeira metade daquela década a população jovem crescia a uma média anual próxima a 3,3\%, nos anos 90 seu crescimento havia declinado para 1,4\% ao ano. A par dessa tendência, a proporção de jovens na população total latino-americana reduziu-se levemente, passando de 20,1\% em 1980 para 19,5\% em 2000 (DIEZ DE MEDINA, 2001, p. 18).

Conforme aludido anteriormente, o Brasil encontra-se em plena transição demográfica, com ritmo de crescimento populacional em declínio. Nos anos 70 , a população do país crescia a uma média anual de $2,5 \%$, diminuindo para $1,9 \%$ na década de 80 e para 1,6\% na de 90 (IBGE, Censos Demográficos, 1970, 1980, 1991 e 2000). Já a evolução do segmento jovem apresentou, neste mesmo período, diferenças importantes em relação à da população total: seu ritmo de crescimento caiu mais intensamente entre as décadas de 70 e 80 (de 3,0\% ao ano para 1,2\% ano), mas voltou a se elevar na de 90 (para 2,0\% ao ano) (IBGE, Censos Demográficos, $1970,1980,1991$ e 2000). Com isso, a proporção de jovens sobre a população total do país, que havia declinado de $21,1 \%$ para $19,5 \%$, entre 1980 e 1991, registrou pequena elevação para 20,1\%, em 2000 (IBGE, Censos Demográficos 1980, 1991 e 2000).

Quando se abordam os efeitos do crescimento populacional sobre a situação dos jovens no mercado de trabalho, um fenômeno relevante é o das descontinuidades demográficas (BERCOVICH e MADEIRA, 1990; MUNIZ, 2002; BERCOVICH e MASSÉ, 2004). Tais descontinuidades caracterizamse pelo crescimento desigual dos diferentes grupos etários ao longo do tempo e por mudanças bruscas no tamanho de coortes sucessivas (BERCOVICH e MADEIRA, 1990, p. 610). De particular interesse para o trabalho que está sendo ora desenvolvido, Bercovich e Madeira (1990, p. 619) identificam dois momentos que seriam representativos desse fenômeno no país, por elas denominado de onda jovem: o primeiro, mais intenso, observado entre 1965 e 1980, e o segundo, mais tênue, ocorrido de 1990 a 2000. Em ambos os períodos, portanto, esperar-se-iam condições de maior adversidade para os jovens no mercado de trabalho, devido à expansão do tamanho da sua coorte.

Caberia ainda destacar que as descontinuidades demográficas identificadas pelo estudo de Bercovich e Madeira (1990), para o caso brasileiro, constituem um fenômeno eminentemente urbano, sendo observadas com maior intensidade nas regiões mais desenvolvidas do país. Isto indica que elas possuem também vínculos com processos migratórios para as áreas urbanas e relativamente mais desenvolvidas.

O estudo de Muniz (2002) buscou combinar o fenômeno das descontinuidades demográficas com a hipótese da expansão da coorte juvenil para analisar a situação desse grupo populacional no mercado de trabalho metropolitano brasileiro. Este trabalho focalizou as seis regiões metropolitanas do país em que é realizada a Pesquisa Mensal de Emprego (PME) do IBGE, utilizando-se uma série de dados anuais cuja cobertura era a do período 1982-2000. A par destes aspectos, o estudo desagregou a população jovem por faixas etárias (15 a 19 e 20 a 24 anos) e sexo. Quanto aos resultados obtidos por esse trabalho, o efeito do tamanho relativo da 
coorte de jovens sobre a taxa de ocupação deste grupo populacional apresentou o sinal esperado (negativo) e era estatisticamente significativo, tanto para os jovens de 15 a 19 anos quanto para os de 20 a 24 anos, assim como para ambos os sexos, confirmando plenamente a hipótese que estava sendo testada. Já na especificação que utilizava a taxa de desemprego ${ }^{5}$ dos jovens como variável dependente, os resultados encontrados no estudo foram menos conclusivos: para os jovens de 20 a 24 anos, o efeito do tamanho relativo da coorte juvenil evidenciava o sinal esperado (positivo), mas era estatisticamente significativo somente para os homens; no caso daqueles de 15 a 19 anos, o efeito do tamanho relativo da coorte juvenil possuía o sinal negativo para ambos os sexos, implicando a rejeição da hipótese do efeito do tamanho relativo da coorte sobre a taxa de desemprego dos jovens desta faixa etária.

$\mathrm{Na}$ próxima seção deste estudo, a hipótese da expansão da coorte será retomada e testada para avaliar a situação dos jovens no mercado de trabalho da RMPA, particularmente no que se refere às taxas de ocupação e de desemprego desse grupo populacional.

\section{Tamanho da coorte, ocupação e desemprego dos jovens: a experiência recente da RMPA}

O propósito desta seção é testar a hipótese do efeito do tamanho da coorte juvenil sobre a ocupação e o desemprego desse grupo populacional na RMPA. Para tanto, o estudo vale-se da base de dados da Pesquisa de Emprego e Desemprego (PED-RMPA), sendo o período de cobertura do trabalho o de 1993 a $2004 .^{6}$

Tendências da população juvenil e de sua inserção no mercado de trabalho da $R M P A^{7}$

A população jovem na RMPA aumentou de 485 mil para 630 mil pessoas, entre 1993 e 2004 (Gráfico 1). Em termos de sua composição por sexo, constata-se que o contingente de mulheres jovens, que era levemente superior ao de homens jovens em 1993, havia sido superado ao final do período: em 2004, o segmento masculino correspondia a 319 mil indivíduos, enquanto o feminino equivalia a 311 mil. Não obstante esta mudança na composição por

GRÁFICO 1

População jovem, por sexo

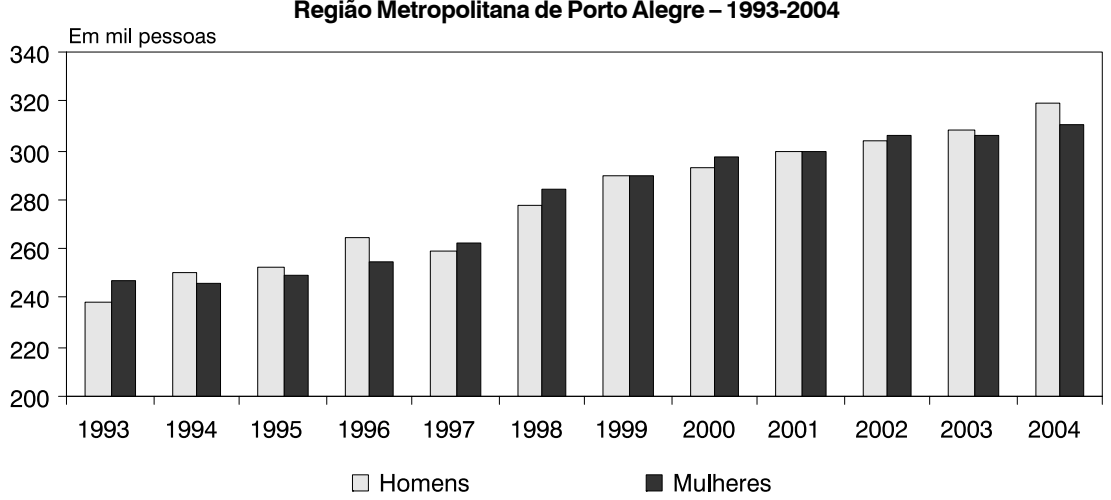

Fonte: PED-RMPA - Convênio FEE, FGTAS/Sine-RS, Seade-SP, Dieese e apoio PMPA.

\footnotetext{
${ }^{5}$ É interessante perceber que neste estudo a taxa de desemprego dos jovens é definida através da relação entre o número de desempregados e a população deste grupo etário, o que não é usual em estudos do mercado de trabalho, pois este indicador costuma ser medido pela relação entre o número de desempregados e a população economicamente ativa (MUNIZ, 2002, p. 83). ${ }^{6}$ O período de análise inicia em 1993 porque este é o primeiro ano para o qual se dispõe de médias anuais dos dados da PED-RMPA ${ }^{7}$ Nesta seção, o grupo populacional jovem foi delimitado como correspondendo à faixa etária de 16 a 24 anos. Adota-se esta faixa etária - e não a utilizada pela Organização das Nações Unidas - porque a idade mínima de ingresso legal no mercado de trabalho do país é de 16 anos.
} 
sexo da população juvenil na RMPA, em que os homens jovens passaram a ser majoritários, a participação de cada um dos sexos na população juvenil mantinha-se bastante próxima ao final do período, configurando uma situação praticamente paritária entre ambos.

Entre 1993 e 2004, o ritmo de crescimento do segmento jovem, com uma taxa média anual de $2,4 \%$, foi superior ao da população total na RMPA, com $1,6 \%$ ao ano (Gráfico 2). Essa diferença de ritmo de crescimento entre a população jovem e a total pode ter contribuído para a ocorrência de uma onda jovem na região metropolitana, nos anos 90, conforme identificada pelo estudo de Bercovich e Madeira (1990) para a realidade do país. ${ }^{8}$ No caso específi- co da população jovem, os dados contidos no Gráfico 2 também revelam que os indivíduos de sexo masculino evidenciaram crescimento populacional mais intenso do que os de sexo feminino, diferentemente do que ocorreu entre a população total.

Em face dos comportamentos descritos, houve aumento da proporção de jovens na população total da RMPA, passando de $15,7 \%$, em 1993, para $17,1 \%$, em 2004 (Gráfico 3). Cabe recuperar, como foi visto na seção anterior deste trabalho, que uma tendência semelhante a essa também foi identificada quando da comparação dos Censos Demográficos de 1991 e 2000, pois a proporção de jovens na população total do país também se elevou levemente naquele período.

\section{GRÁFICO 2}

Taxas médias anuais de crescimento da população total e de jovens, por sexo Região Metropolitana de Porto Alegre - 1993/2004

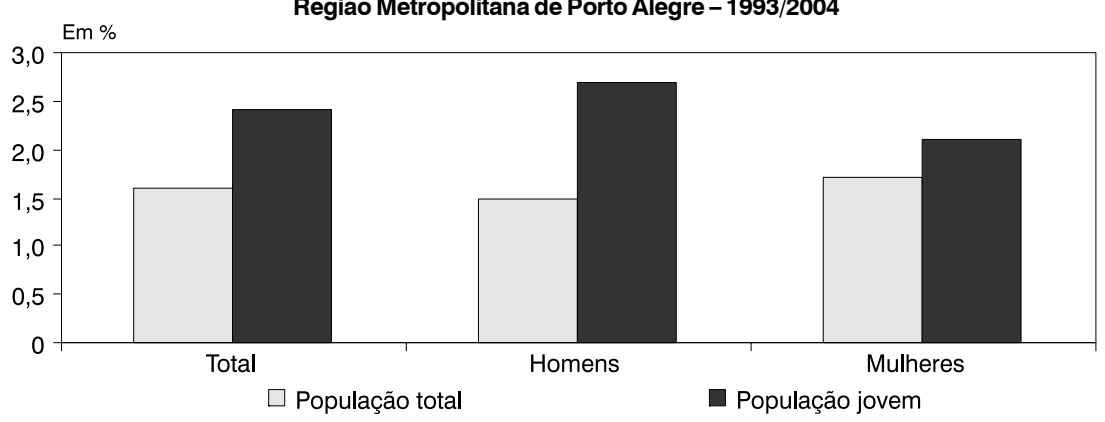

Fonte: PED-RMPA - Convênio FEE, FGTAS/Sine-RS, Seade-SP, Dieese e apoio PMPA.

GRÁFICO 3

Participação de jovens na população total

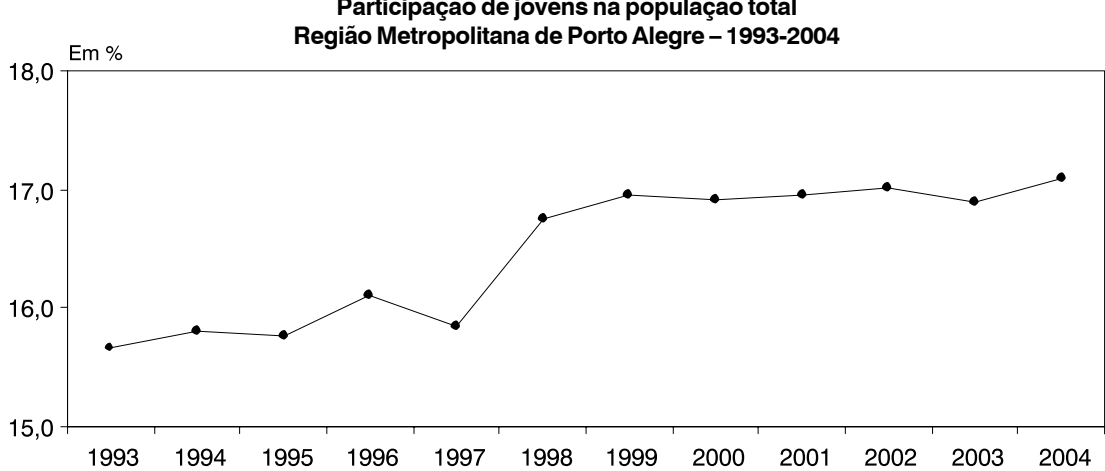

Fonte: PED-RMPA - Convênio FEE, FGTAS/Sine-RS, Seade-SP, Dieese e apoio PMPA.

${ }^{8}$ Nesse período, não se observa tendência de aumento da intensidade do fluxo imigratório de jovens para a RMPA. Assim, tomandose como referência os jovens que haviam imigrado para a região metropolitana em um período de até três anos, a cada ano, estes, que correspondiam a 8,9\% da população jovem total em 1993, haviam recuado para 7,0\% em 2004. 
No âmbito do mercado de trabalho da RMPA, o nível de engajamento da população juvenil na força de trabalho mantevese idêntico quando se comparam o início e o final do período em análise (Gráfico 4). Assim, a taxa de participação dos jovens no mercado de trabalho da região, que era de $70,3 \%$ em 1993, encontrava-se exatamente neste mesmo patamar em 2004.

No que diz respeito à intensidade de engajamento dos jovens em atividades laborais na RMPA, conforme a segmentação por sexo, alguns aspectos necessitam ser destacados (Gráfico 4). Os homens jovens evidenciavam taxas de participação $(74,8 \%)$ mais elevadas do que as mulheres jovens $(65,7 \%)$ no mercado de trabalho da região, em 2004. Todavia, a tendência deste indicador foi discrepante entre estes dois grupos populacionais ao longo do período, pois, enquanto para os homens jovens ocorreu declínio da taxa de participação, para as mulheres jovens o indicador em análise mostrou elevação. Com isso, o diferencial de taxas de participação entre os sexos, que era desfavorável em 21,2 pontos percentuais para as mulheres jovens em 1993, havia se reduzido para 9,1 pontos percentuais em 2004. Esta mudança no padrão de inserção no mercado de trabalho entre os sexos, em que se intensifica o engajamento das mulheres em atividades produtivas, corresponde a um movimento mais amplo que vem ocorrendo no âmbito do mercado de trabalho local, conforme identificaram outros estudos (GALEAZZI et al., 2002; MARQUES et al., 2004).

O nível de ocupação dos jovens apresentou tendência de elevação na RMPA, no período em análise, tendo passado de 268 mil ocupados em 1993 para 313 mil em 2004, o que correspondeu a um crescimento de 16,8\% (Gráfico 5). A análise por sexo identifica que ocorreu tendência de elevação tanto para os homens quanto para as mulheres, mas um pouco mais intensa para o segmento feminino: entre 1993 e 2004, o contingente de mulheres jovens ocupadas aumentou de 111 mil para 133 mil pessoas, com um crescimento de $19,8 \%$, enquanto o de homens jovens ascendeu de 157 mil para 180 mil, com aumento de $14,6 \%$.

Ainda no que se refere à ocupação juvenil na RMPA, sua composição por sexo apresentou pequena alteração entre o início e o final do período em análise, devido à performance ocupacional relativamente melhor do sexo feminino, conforme visto anteriormente. Assim, as mulheres jovens, que correspondiam a $41,4 \%$ da ocupação juvenil da região em 1993, passaram a representar uma proporção levemente superior em 2004 (42,5\%). Já os homens

\section{GRÁFICO 4}

Taxas de participação dos jovens, por sexo Região Metropolitana de Porto Alegre - 1993-2004

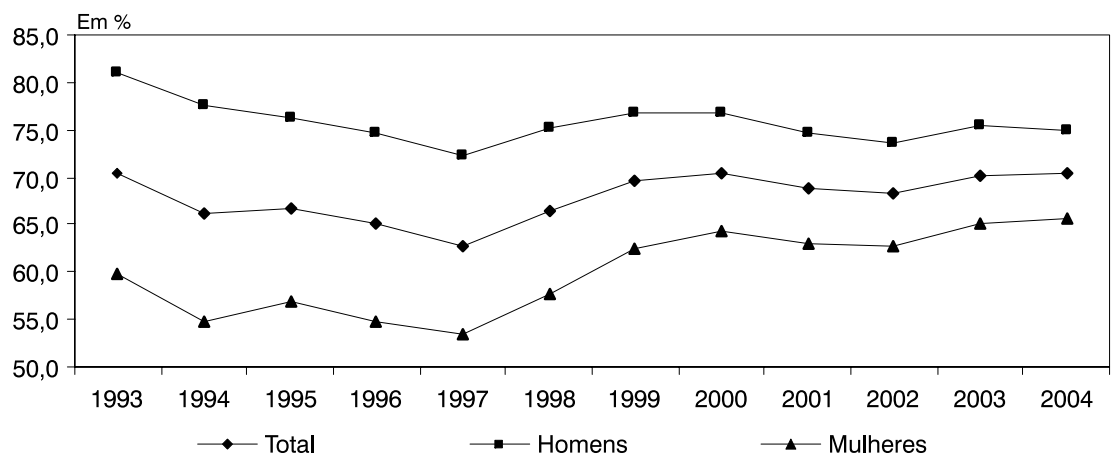

Fonte: PED-RMPA - Convênio FEE, FGTAS/Sine-RS, Seade-SP, Dieese e apoio PMPA. 
GRÁFICO 5

Jovens ocupados, por sexo

Região Metropolitana de Porto Alegre - 1993-2004

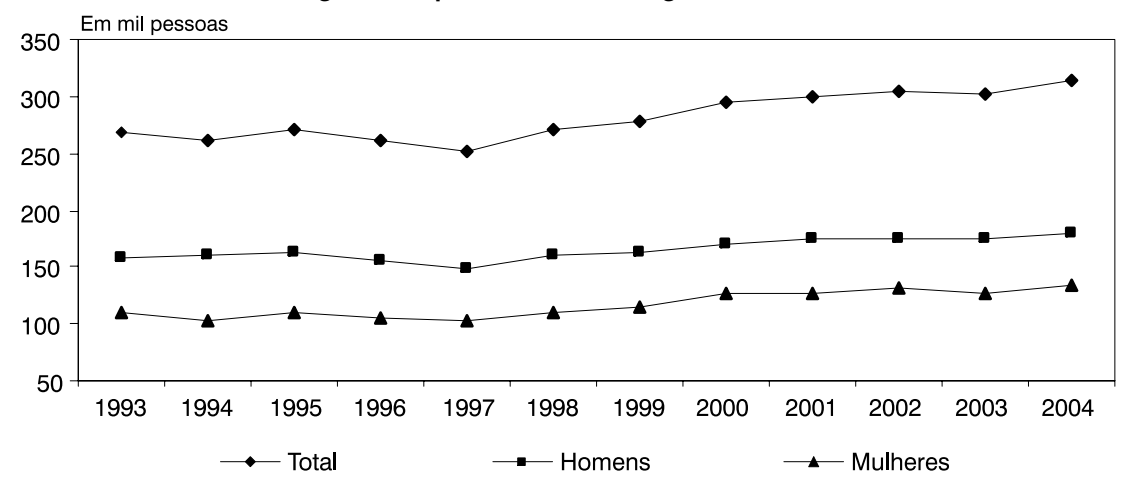

Fonte: PED-RMPA - Convênio FEE, FGTAS/Sine-RS, Seade-SP, Dieese e apoio PMPA.

GRÁFICO 6

Distribuição dos jovens ocupados, por sexo

Região Metropolitana de Porto Alegre - 1993-2004

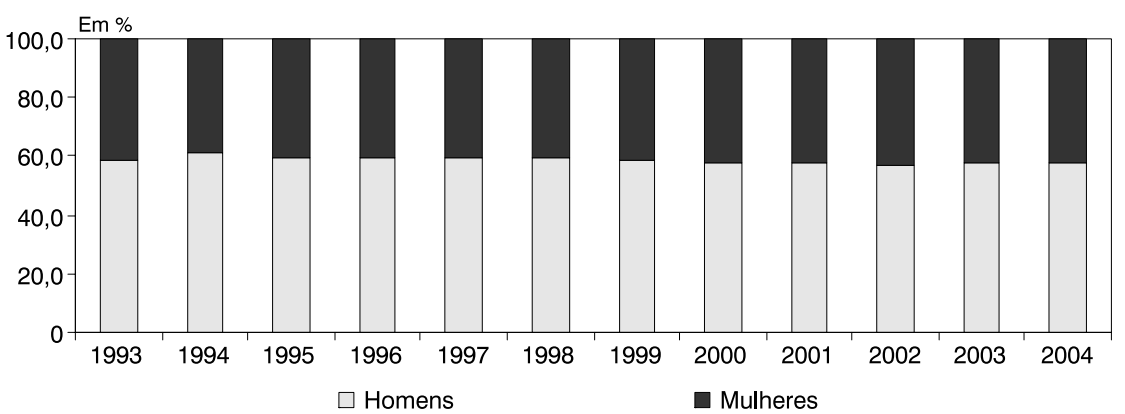

Fonte: PED-RMPA - Convênio FEE, FGTAS/Sine-RS, Seade-SP, Dieese e apoio PMPA.

jovens, como decorrência, recuaram de 58,6\% na ocupação juvenil em 1993 para 57,5\% em 2004 (Gráfico 6), permanecendo, não obstante esta pequena mudança, claramente majoritários entre os ocupados deste grupo populacional na RMPA.

Uma das características mais marcantes da inserção dos jovens no mercado de trabalho é a elevada incidência do desemprego, conforme identificam muitos estudos (O'HIGGINS, 1997; OIT, 2000 e 2004; POCHMANN, 2000; TOKMAN, 2003). No caso da RMPA, constata-se que a taxa de desemprego juvenil não só é muito elevada, como também apresentou aumento acentuado ao longo do período, passando de 21,4\% em 1993 para 29,3\% em 2004 - ou seja, neste último ano, praticamente 30 em cada 100 jovens que estavam no mercado de trabalho metropolitano se encontravam na situação de desemprego (Gráfico 7). Pode-se também constatar, observando-se todo o período, que ocorreram dois momentos em que o desemprego juvenil se atenuou: imediatamente após a implementação do Plano Real (1994 e 1995); e posteriormente à desvalorização cambial de 1999 (20002002).

No que diz respeito à taxa de desemprego juvenil por sexo, observou-se tendência semelhante de elevação deste indicador para homens e mulheres na RMPA, no período analisado (Gráfico 7). A par deste aspecto, destaca-se que as mulheres jovens evidenciavam maior incidência do desemprego do que os homens jovens, sendo suas taxas de 
GRÁFICO 7

Taxas de desemprego dos jovens, por sexo

Região Metropolitana de Porto Alegre - 1993-2004

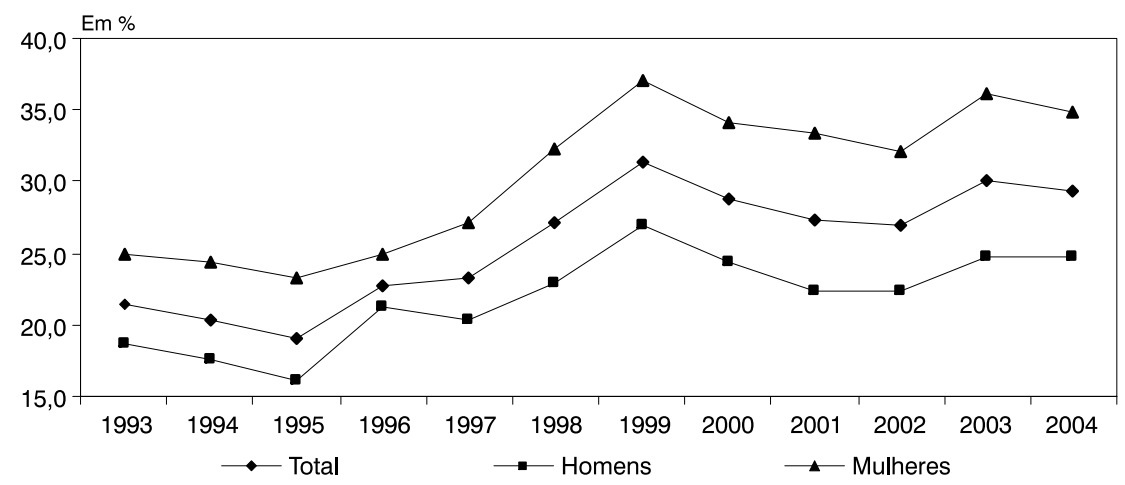

Fonte: PED-RMPA - Convênio FEE, FGTAS/Sine-RS, Seade-SP, Dieese e apoio PMPA.

GRÁFICO 8

Distribuição dos jovens desempregados, por sexo

Região Metropolitana de Porto Alegre - 1993-2004

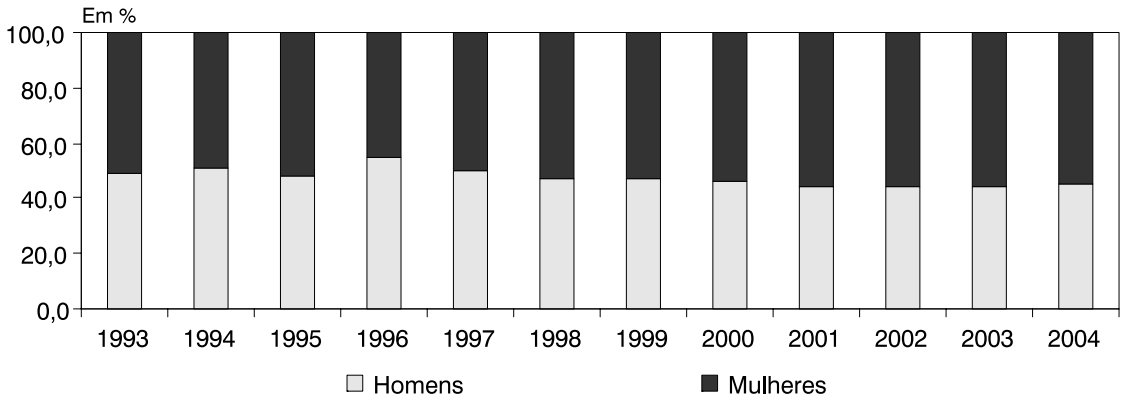

Fonte: PED-RMPA - Convênio FEE, FGTAS/Sine-RS, Seade-SP, Dieese e apoio PMPA.

desemprego em 2004 de $34,8 \%$ e $24,7 \%$, respectivamente. Deve-se ter presente que esta maior incidência do desemprego sobre as mulheres jovens constitui um padrão no caso latino-americano (OIT, 2000), o qual se confirma plenamente nas diversas regiões metropolitanas do Brasil (DIEESE, 2001, cap. 6; MUNIZ, 2002). Pode-se também perceber que ocorreu uma deterioração relativa do desemprego entre as mulheres jovens em comparação aos homens jovens no mercado de trabalho metropolitano, pois o diferencial desfavorável às primeiras de incidência do desemprego elevou-se de 6,3 pontos percentuais em 1993 para 10,1 em 2004.

A combinação dos movimentos descritos neste trabalho, de elevação do engajamento das mulheres jovens no mercado de trabalho e de redução no caso dos homens jovens, por um lado, e de aumento mais acentuado do desemprego entre as mulheres jovens em comparação aos homens jovens, por outro, fez com que se ampliasse a proporção de indivíduos de sexo feminino no contingente de jovens desempregados na RMPA (Gráfico 8). Nesse sentido, as mulheres jovens, que representavam $50,7 \%$ dos desempregados desse grupo populacional na região em 1993, haviam atingido 54,6\% em 2004, com a conseqüente redução da proporção de homens desempregados, de $49,3 \%$ para $45,4 \%$ nesses mesmos anos.

A trajetória ascendente do desemprego juvenil na RMPA está vinculada a uma 
GRÁFICO 9

Taxas médias anuais de crescimento da PEA, da ocupação e do desemprego dos jovens, por sexo Região Metropolitana de Porto Alegre - 1993/2004

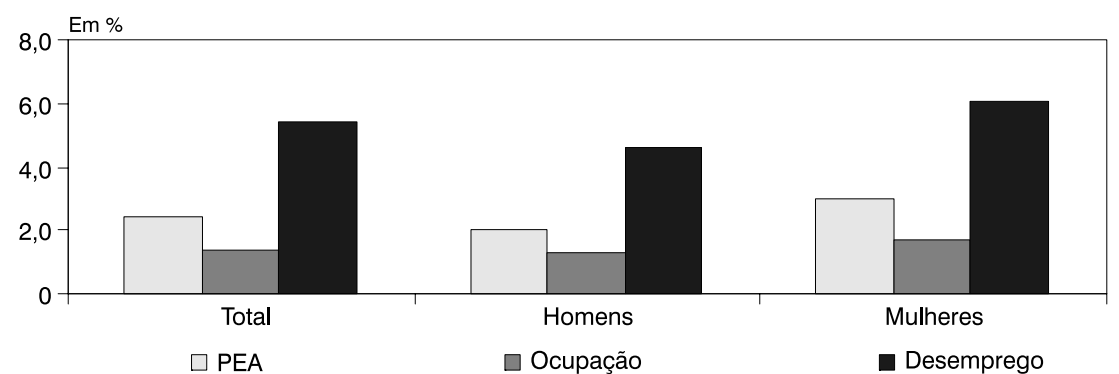

Fonte: PED-RMPA - Convênio FEE, FGTAS/Sine-RS, Seade-SP, Dieese e apoio PMPA.

capacidade relativamente modesta de absorção de mão-de-obra pelo mercado de trabalho local, em decorrência do baixo dinamismo econômico do período. Esta compreensão encontra respaldo no Gráfico 9 , no qual são cotejadas as taxas médias anuais de crescimento da população economicamente ativa (PEA), da ocupação e do contingente de jovens desempregados na RMPA, no período 1993-2004. Conforme pode-se constatar, a PEA juvenil evidenciou crescimento de $2,4 \%$ ao ano, bastante superior ao da ocupação juvenil, de $1,4 \%$ ao ano, com o que o desemprego entre os jovens se elevou a uma taxa média anual muito superior, de 5,4\%. Quando se contrasta o comportamento destas variáveis, segundo sexo, verifica-se que as mulheres jovens tiveram uma performance relativamente melhor do que os homens jovens em termos de crescimento da ocupação. Não obstante, como a PEA elevou-se bem mais entre as mulheres jovens do que entre os homens jovens, isto implicou um ritmo de crescimento mais acelerado do contingente de mulheres jovens desempregadas $(6,1 \%$ ao ano), comparativamente ao de homens jovens desempregados (4,6\% ao ano), deteriorando a situação relativa das primeiras no mercado de trabalho metropolitano.
A estimação do efeito do tamanho relativo da coorte sobre a ocupação e o desemprego dos jovens na RMPA

A partir da compreensão de que ocorreu uma onda jovem no país e em suas regiões metropolitanas nos anos 90 , procura-se estimar os efeitos do tamanho relativo da coorte juvenil sobre a ocupação e o desemprego desse grupo populacional na RMPA.

Considerando este propósito, foram especificados dois modelos econométricos para estimar os efeitos do tamanho relativo da coorte juvenil. ${ }^{9}$

Modelo 1

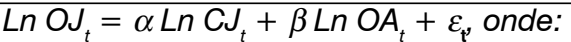
$O J_{t}$ : taxa de ocupação dos jovens no ano $\mathrm{t}$, definida pela divisão do contingente de ocupados deste grupo populacional pela sua respectiva PEA;

$\mathrm{CJ}_{t}$ : tamanho relativo da coorte juvenil no ano $t$, definido pela divisão da população juvenil pela população adulta;

$O A_{t}$ : taxa de ocupação dos adultos no ano $\mathrm{t}$, definida pela divisão do contingente de ocupados deste grupo populacional pela sua respectiva PEA.

Observação: as variáveis foram transformadas em logaritmos naturais.

${ }^{9}$ Os modelos foram especificados de maneira semelhante aos de Korenman e Neumark (1997), O’Higgins (1997) e Muniz (2002). 
Modelo 2

$L n D J_{t}=\alpha L n C J_{t}+\beta L n D A_{t}+\varepsilon_{t}$, onde: $D J_{t}$ : taxa de desemprego dos jovens no ano $t$, definida pela divisão do contingente de desempregados deste grupo populacional pela sua respectiva PEA;

$C J_{t}$ : tamanho relativo da coorte juvenil no ano $t$, definido pela divisão da população juvenil pela população adulta;

$D A_{t}$ : taxa de desemprego dos adultos no ano $t$, definida pela divisão do contingente de desempregados deste grupo populacional pela sua respectiva PEA.

Observação: as variáveis foram transformadas em logaritmos naturais.

No que diz respeito ao Modelo 1, a hipótese é de que o tamanho relativo da coorte juvenil exerça um efeito negativo sobre a taxa de ocupação dos jovens, ou seja, um aumento da coorte implicará uma redução da taxa de ocupação juvenil. Quanto ao Modelo 2, a hipótese é de que o tamanho relativo da coorte juvenil tenha um efeito positivo sobre a taxa de desemprego deste grupo populacional, no sentido de que o seu aumento elevará o desemprego entre os jovens.

Conforme pode-se também observar, existe uma segunda variável explicativa tanto no Modelo 1 quanto no Modelo 2, que são a taxa de ocupação e a de desemprego dos adultos, respectivamente. Seguindo a orientação de outros trabalhos sobre este tema (KORENMAN e NEUMARK, 1997; O'HIGGINS,
1997; MUNIZ, 2002), a inclusão destas variáveis explicativas nos dois modelos tem o propósito de capturar o efeito da demanda agregada da economia sobre as taxas de ocupação e de desemprego dos jovens, constituindo-se, portanto, em proxies que procuram controlar os efeitos do nível de atividade econômica sobre as variáveis dependentes.

Nesse sentido, espera-se, no Modelo 1, que o aumento da taxa de ocupação dos adultos implique elevação da taxa de ocupação dos jovens, pois a economia se encontraria, nestas condições, com maior nível de atividade. Já no Modelo 2, um aumento da taxa de desemprego dos adultos deverá implicar uma elevação da taxa de desemprego dos jovens, pois a economia, supostamente, estaria com um nível de atividade mais reduzido. Por último, cabe destacar que ambos os modelos foram estimados para o total da população jovem e por sexo, com o propósito de tentar identificar se existem efeitos diferenciados do tamanho relativo da coorte juvenil sobre os homens jovens em comparação às mulheres jovens no mercado de trabalho metropolitano.

Os resultados básicos da estimação do Modelo 1 podem ser observados na Tabela 1. Tomando-se a população jovem da RMPA como um todo, constata-se que a estimativa do coeficiente do tamanho relativo da coorte juvenil $(-0,902)$ tem o sinal esperado, sendo estatisticamente significativa ao nível de $5 \%$, o que sugere a confirmação da hipótese que está sendo testada. É interessante

TABELA 1

Resultados básicos da estimação do Modelo 1

\begin{tabular}{|c|c|c|c|}
\hline \multirow{2}{*}{ Variável dependente } & \multicolumn{2}{|c|}{ Variáveis independentes } & \multirow{2}{*}{$\mathbf{R}^{2}$} \\
\hline & $C J_{t}$ & $\mathbf{O A}_{t}$ & \\
\hline \multicolumn{4}{|l|}{ Jovens } \\
\hline$O J_{t}$ & $\begin{array}{c}-0,902 \text { * } \\
(-4,0)\end{array}$ & $\begin{array}{c}1,639 * * \\
(9,63)\end{array}$ & 0,845 \\
\hline \multicolumn{4}{|l|}{ Homens jovens } \\
\hline $\mathrm{OJ}_{\mathrm{t}}$ & $\begin{array}{c}-0,515 \text { * } \\
(-3,72)\end{array}$ & $\begin{array}{c}1,358 * * \\
(12,99)\end{array}$ & 0,895 \\
\hline \multicolumn{4}{|l|}{ Mulheres jovens } \\
\hline $\mathrm{OJ}_{\mathrm{t}}$ & $\begin{array}{c}-1,354 \text { * } \\
(-3,77)\end{array}$ & $\begin{array}{c}1,965 * * \\
(7,24)\end{array}$ & 0,776 \\
\hline
\end{tabular}

Fonte: PED-RMPA - Convênio FEE, FGTAS/Sine-RS, Seade-SP, Dieese e apoio PMPA.

Nota: Método de estimação: mínimos quadrados ordinários.

Tamanho da amostra: $\mathrm{n}=12$

Estatísticas $t$ entre parênteses.

A notação ** corresponde ao nível de significância de $1 \%$ e * ao de $5 \%$. 
também destacar o quanto é importante a situação macroeconômica para a determinação do nível ocupacional dos jovens, o que é capturado no modelo pela estimativa do coeficiente da taxa de ocupação dos adultos $(1,639)$, estatisticamente significativa ao nível de 1\%: ou seja, trata-se de um efeito de magnitude superior ao do tamanho relativo da coorte juvenil. No que se refere à população juvenil como um todo e à estimação do Modelo 1, caberia ainda assinalar que o seu poder de explicação é bastante elevado, pois o coeficiente de determinação é de 0,845.

No que diz respeito ao Modelo 1, passase agora a apresentar os resultados da sua estimação para a população juvenil da RMPA, segundo sexo (Tabela 1). As estimativas dos coeficientes do tamanho relativo da coorte juvenil têm o sinal esperado (negativo) tanto para homens como para mulheres, sendo estatisticamente significativas ao nível de $5 \%$, o que corrobora a hipótese central deste estudo. É interessante destacar, não obstante, que o efeito do tamanho relativo da coorte juvenil é bem mais acentuado no caso das mulheres jovens em comparação ao dos homens jovens, o que sugere uma condição de maior fragilidade entre as primeiras no mercado de trabalho metropolitano.

Quanto ao efeito exercido pela taxa de ocupação dos adultos, cujo papel no modelo é o de capturar o nível de atividade econômica, pode-se destacar os seguintes aspectos: tanto entre os homens jovens quanto entre as mulheres jovens, as estimativas dos coeficientes desta variável têm o sinal esperado (positivo), sendo ambas estatisticamente significativas ao nível de 1\%; o efeito estimado desta variável explicativa sobre a taxa de ocupação de cada um dos sexos também é de maior magnitude do que o do tamanho relativo da coorte juvenil; e, dado que a estimativa do efeito da taxa de ocupação dos adultos é maior na regressão relativa às mulheres jovens, se depreende que estas são atingidas mais intensamente pelas flutuações do nível de atividade econômica. Finalmente, quanto ao poder de explicação do Modelo 1 para cada um dos sexos, registre-se que o coeficiente de determinação na regressão relativa aos homens jovens $(0,895)$ é mais elevado do que o da regressão relativa às mulheres jovens $(0,776)$.

Os resultados básicos da estimação do Modelo 2 podem ser conhecidos por meio da Tabela 2. Iniciando pela apresentação dos resultados da regressão relativa ao total da população jovem na RMPA, constata-se que a estimativa do coeficiente do tamanho relativo da coorte juvenil $(0,435)$ tem o sinal esperado, sendo estatisticamente significativa ao nível de $1 \%$. Também no Modelo 2 a situação macroeconômica, apreendida pela taxa de desemprego da população adulta, tem um efeito de maior magnitude sobre a taxa de desemprego dos jovens: o coeficiente estimado desta variável é de 0,755 , estatisticamente significativo ao nível de $1 \%$. Assinale-se que também neste caso o poder de explicação do modelo é elevado, pois o coeficiente de determinação é de 0,924 .

No que diz respeito à estimação do Modelo 2, seus resultados, quando se efetua a segmentação da população jovem por sexo (Tabela 2), mostram que a estimativa do coeficiente do tamanho relativo da coorte juvenil tem o sinal esperado (positivo), sendo estatisticamente significativa ao nível de $1 \%$ para ambos os sexos. Caberia destacar que, no caso em análise, embora persista uma diferença desfavorável às mulheres jovens em relação aos homens jovens, esta é relativamente menor do que a observada no Modelo 1.

Quanto às estimativas do efeito do nível de atividade econômica sobre as taxas de desemprego dos jovens, por sexo, apreendidas no Modelo 2 pela taxa de desemprego dos adultos, para ambos os sexos estas têm o sinal esperado (positivo), sendo estatisticamente significativas ao nível de $1 \%$. Também neste caso, o efeito estimado desta variável explicativa é de magnitude superior ao do tamanho relativo da coorte juvenil. Embora apresentando uma menor diferença, aqui se reafirma uma situação relativamente desfavorável para as mulheres jovens, pois estas são mais atingidas por 
TABELA 2

Resultados básicos da estimação do Modelo 2

\begin{tabular}{|c|c|c|c|}
\hline \multirow{2}{*}{ Variável dependente } & \multicolumn{2}{|c|}{ Variáveis independentes } & \multirow{2}{*}{$\mathbf{R}^{2}$} \\
\hline & $\mathbf{C J}_{\mathrm{t}}$ & $\mathrm{DA}_{t}$ & \\
\hline \multicolumn{4}{|l|}{ Jovens } \\
\hline \multirow[t]{2}{*}{$\mathrm{DJ}_{\mathrm{t}}$} & $0,435 * \star$ & $0,755 * *$ & 0,924 \\
\hline & $(8,86)$ & $(10,52)$ & \\
\hline \multicolumn{4}{|l|}{ Homens jovens } \\
\hline \multirow[t]{2}{*}{$D J_{t}$} & $0,421 * *$ & $0,708 * *$ & 0,941 \\
\hline & $(10,47)$ & $(12,06)$ & \\
\hline \multicolumn{4}{|l|}{ Mulheres jovens } \\
\hline \multirow[t]{2}{*}{$D J_{t}$} & $0,487 * \star$ & $0,753 * *$ & 0,861 \\
\hline & $(7,03)$ & $(7,44)$ & \\
\hline
\end{tabular}

Fonte: PED-RMPA - Convênio FEE, FGTAS/Sine-RS, Seade-SP, Dieese e apoio PMPA.

Nota: Método de estimação: mínimos quadrados ordinários.

Tamanho da amostra: $\mathrm{n}=12$.

Estatísticas $t$ entre parênteses.

A notação ** corresponde ao nível de significância de $1 \%$.

uma conjuntura macroeconômica adversa, capturada pela taxa de desemprego dos adultos. Por último, é importante registrar que o poder de explicação das regressões relativas a ambos os sexos é elevado, ainda que no caso dos homens jovens o coeficiente de determinação $(0,941)$ seja superior ao das mulheres jovens $(0,861)$.

Em síntese, os resultados da estimação dos modelos indicam a confirmação da hipótese que organiza este trabalho, pois foram encontrados os efeitos previstos do tamanho relativo da coorte juvenil sobre a ocupação e o desemprego deste grupo populacional na RMPA, no período enfocado. Não obstante, a estimação dos modelos também chamou a atenção para a importância do nível de atividade econômica para a situação dos jovens no mercado de trabalho, cujos efeitos são até mesmo de maior magnitude sobre a ocupação e o desemprego. Tal resultado, assinale-se, vai ao encontro do que revelaram trabalhos abordando esta mesma questão nos países da OCDE e nas regiões metropolitanas brasileiras. $^{10}$

\section{Considerações finais}

Este estudo procurou analisar os efeitos do tamanho relativo da coorte juvenil sobre a ocupação e o desemprego deste grupo populacional na RMPA, no período 1993-2004. Conforme foi mostrado no trabalho, o contingente de jovens cresceu em ritmo mais intenso do que a população total da RMPA no período em foco, o que se vincula à ocorrência de uma onda jovem no país na década de 90 , com ênfase especial em suas áreas metropolitanas. Esse fenômeno teve como conseqüência uma elevação da proporção de jovens na população total da RMPA, entre 1993 e 2004.

No âmbito do mercado de trabalho da RMPA, embora a taxa de participação dos jovens não tenha permanecido constante ao longo do período, quando se compararam o seu início e o seu final, verificou-se que este indicador manteve-se exatamente no mesmo patamar. Por sua vez, foram distintas as evoluções do grau de engajamento no mercado de trabalho dos homens jovens em relação às mulheres jovens: enquanto os primeiros apresentaram tendência de declínio em sua taxa de participação, as últimas evidenciaram tendência de elevação, reduzindo-se, assim, o diferencial de engajamento na força de trabalho entre ambos.

A ocupação juvenil registrou crescimento relativamente modesto na RMPA no período, revelando uma baixa capacidade

\footnotetext{
${ }^{10} \mathrm{~A}$ esse respeito, ver, para os países da OCDE, Korenman e Neumark (1997) e O'Higgins (1997) e, para as regiões metropolitanas do Brasil, Muniz (2002).
} 
de absorção de mão-de-obra pela economia local. Dado que este crescimento foi amplamente superado pelo da força de trabalho deste grupo populacional, isto implicou um agravamento do desemprego entre os jovens, o qual cresceu em ritmo muito mais acelerado no período. Cabe mencionar que as mulheres jovens tiveram desempenho, em termos de crescimento do nível de ocupação, levemente superior ao dos homens jovens. Não obstante, como a força de trabalho feminina cresceu em ritmo mais acelerado do que a masculina, isto implicou aumento muito mais intenso do desemprego entre as mulheres jovens, com o que a sua situação relativa se deteriorou no mercado de trabalho metropolitano.

Os resultados da estimação dos modelos econométricos indicaram a confirmação da hipótese do efeito do tamanho relativo da coorte juvenil sobre a ocupação e o desemprego deste grupo populacional, no mercado de trabalho da RMPA. O efeito do tamanho relativo da coorte juvenil mostrou-

\section{Referências bibliográficas}

BASTOS, R. O segmento juvenil do mercado de trabalho da Região Metropolitana de Porto Alegre: um estudo com ênfase na escolaridade. Ensaios FEE, Porto Alegre, FEE, v. 26, número especial, p. 271-298, 2005.

BERCOVICH, A.; MADEIRA, F. Descontinuidades demográficas no Brasil e no Estado de São Paulo. In: ENCONTRO NACIONAL DE ESTUDOS POPULACIONAIS, 7, Caxambu, 1990. Anais... Belo Horizonte: Abep, v. 2, 1990, p. 595-631.

BERCOVICH, A.; MASSÉ, G. Descontinuidades demográficas, ondas jovens e mercado de trabalho: uma comparação entre Brasil e Argentina. In: CONGRESSO DA ASSOCIAÇÃO LATINO AMERICANA DE POPULAÇÃO, 1, Caxambu, 2004. Anais... Caxambu: Alap, 2004.

COMISIÓN ECONÓMICA PARA AMÉRICA LATINA Y EL CARIBE. La juventude en Iberoamérica: tendencias y urgencias. Santiago: Cepal, 2004. se de maior magnitude no caso da taxa de ocupação, demonstrando uma maior sensibilidade desta em comparação à taxa de desemprego, o que remeteria à necessidade de estudos posteriores, para que sua causa fosse plenamente conhecida. No que se refere à segmentação da força de trabalho juvenil por sexo, os resultados da estimação dos modelos indicaram que as mulheres jovens são mais intensamente afetadas pelo efeito do tamanho relativo da coorte juvenil do que os homens jovens, respaldando o entendimento de que os indivíduos de sexo feminino se encontram em situação de maior fragilidade no mercado de trabalho metropolitano. Por último, em consonância com outras pesquisas, a estimação dos modelos indicou que o nível de atividade econômica tem efeitos de maior magnitude sobre a ocupação e o desemprego dos jovens, o que reforça a compreensão de que a performance macroeconômica é de grande relevância para as perspectivas deste grupo populacional no mercado de trabalho.

DIEESE - Departamento Intersindical de Estatística e Estudos Sócio-Econômicos. A situação do trabalho no Brasil. São Paulo: Dieese, 2001.

DIEZ DE MEDINA, R. Jóvenes y empleo en Ios anos 90. Montevideo: OIT/Cinterfor, 2001.

GALEAZZI, I. et al. Desemprego e precarização do trabalho: a experiência na Região Metropolitana de Porto Alegre. In: WILTGEN, R.; GARCIA, L. (Coords.). Transformações do mercado de trabalho metropolitano: os 10 anos da PED-RMPA. Porto Alegre: FEE, FGTAS/Sine-RS, Dieese, Seade-SP, FAT/ MTE, PMPA, 2002.

IBGE - Instituto Brasileiro de Geografia e Estatística. Censo demográfico de 1970. Rio de Janeiro: IBGE, 1971.

Censo demográfico de 1980. Rio de Janeiro: IBGE, 1980.

Censo demográfico de 1991. Rio de Janeiro: IBGE, 1991. 
. Censo demográfico de 2000. Rio de Janeiro: IBGE, 2000.

KORENMAN, S.; NEUMARK, D. Cohort crowding and youth labor markets: a crossnational analysis. Cambridge: NBER, 1997. (Working paper, 6031).

MARQUES, E. et al. Conjunturas desfavoráveis consolidam o perfil feminino do desemprego na Região Metropolitana de Porto Alegre. Mulher e Trabalho, Porto Alegre, FEE, FGTAS/Sine-RS, Dieese, Seade-SP, FAT/MTE e apoio PMPA, v. 4, p. 9-28, 2004.

MUNIZ, J. As descontinuidades demográficas exercem efeitos sobre o mercado de trabalho metropolitano dos jovens? Revista Brasileira de Estudos de População, v. 19, n. 2, p. 65-97, 2002.
O'HIGGINS, N. The challenge of youth unemployment. Genebra: OIT, 1997. (Employment and training papers, 7).

ORGANIZACIÓN INTERNACIONAL DEL TRABAJO. Emplear a los jóvenes: promover un crecimiento intensivo en empleo. Genebra: OIT, 2000.

Tendencias mundiales del empleo juvenil. Genebra: OIT, 2004.

POCHMANN, M. A batalha pelo primeiro emprego. São Paulo: Publisher Brasil, 2000.

TOKMAN, V. Desempleo juvenil en el cono sur: causas, consecuencias y políticas. Santiago: Fundación Friedrich Ebert, 2003.

UNITED NATIONS. World youth report 2003. Nova lorque: UN, 2003.

\section{Abstract}

Population growth, labor by youth and unemployment: the recent experience of the Metropolitan Area of Porto Alegre, Brazil

This article studies to what extent the relative size of the young population in the Metropolitan Area of Porto Alegre (RMPA) affected employment and unemployment of this group between 1993 and 2004. This question has taken on considerable importance, based on two different factors. The first is the fact that a number of studies have indicated the occurrence of the phenomenon of a wave of youth in the largest metropolitan areas in the country during the 1990s. Secondly, the concomitant trend toward higher unemployment among youth during this same period. The article is organized in the following way: after the Introduction, Section 2 presents a synthesis of the arguments regarding the effects of the relative size of the cohort of youth on the situation of this group in the labor market. Section 3 identifies the main trends of the younger population within the labor market in the RMPA and estimates the effects of the relative size of this cohort on the employment and unemployment of this population group. Finally, the main conclusions of the study are presented.

Key words: Youth cohort. Youth employment. Youth unemployment. Metropolitan labor market.

Recebido para publicação em 28/08/2006. Aceito para publicação em 06/11/2006. 
